\title{
Reducing the Effect of Transducer Mount Induced Noise on Aeroacoustic Wind Tunnel Testing Data with a New Transducer Mount Design
}

\author{
Andrew J. Herron ${ }^{1}$, Darren K. Reed ${ }^{1}$, and Donald K. Nance ${ }^{2}$ \\ NASA George C. Marshall Space Flight Center, Huntsville, Alabama, 35812
}

\begin{abstract}
Flight vehicle aeroacoustic environments induced during transonic and supersonic flight are usually predicted by subscale wind tunnel testing utilizing high frequency miniature pressure transducers. In order to minimize noise induced by the measurement itself, transducer flush mounting with the model surface is very important. The National Aeronautics and Space Administration (NASA) has accomplished flushness in recent testing campaigns via use of a transducer holder that can be machined and sanded. A single hole in the holder allows the flow medium to interact with the transducer diaphragm. Noise is induced by the resulting cavity however, and is a challenge to remove in post-processing. A new holder design has been developed that minimizes the effects of this transducer mount induced noise (XMIN) by reducing the resonance amplitude or increasing its resonance frequency beyond the range of interest. This paper describes a test conducted at the NASA/George C. Marshall Space Flight Center Trisonic Wind Tunnel intended to verify the effectiveness of this design. The results from this test show that this new transducer holder design does significantly reduce the influence of XMIN on measured fluctuating pressure levels without degrading a transducer's ability to accurately measure the noise external to the model.
\end{abstract}

\section{Nomenclature}

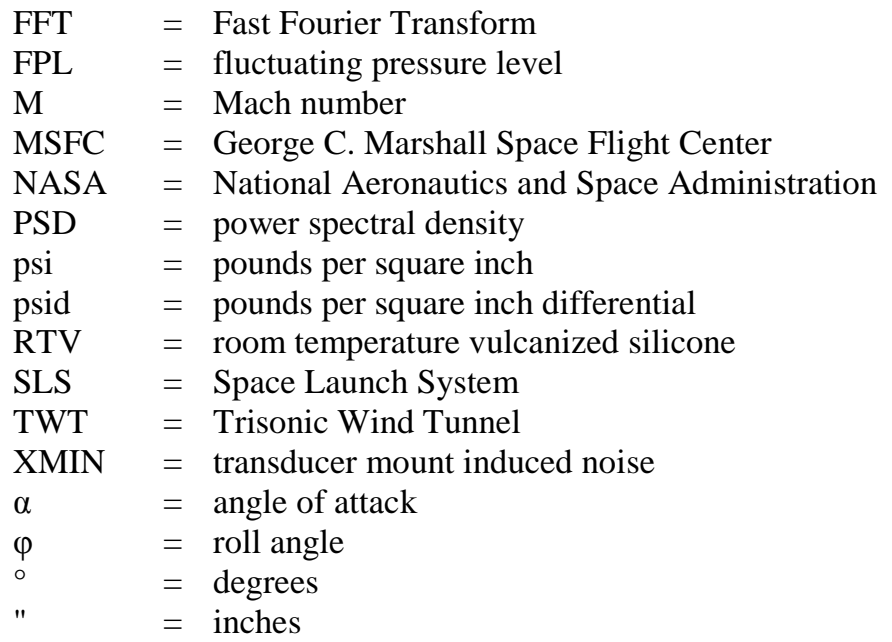

${ }^{1}$ Aerospace Engineer, Aerosciences Branch, EV33.

${ }^{2}$ Aerospace Engineer, Fluid Dynamics Branch, ER42, Member AIAA.

American Institute of Aeronautics and Astronautics 


\section{Introduction}

$\mathrm{C}$ HARACTERIZATION of flight vehicle unsteady aerodynamics is a field usually studied through experimentation, which is often carried out in the form of large scale wind tunnel testing ${ }^{1}$. Measurement of the fluctuating pressures induced by the boundary layer noise in such tests is made with miniature pressure transducers installed in a subscale model of the vehicle of interest ${ }^{2}$. Literature shows that noise levels between two to five decibels (referenced to $20 \times 10^{-6}$ pascals) can be induced when the transducer surface is mounted out of flush with the model outer surface ${ }^{3}$. Because accurate determination of aerodynamically induced acoustic environments are critical for design, this effect must be minimized ${ }^{4}$.

Achieving exact flush mounting is a practical challenge however. If

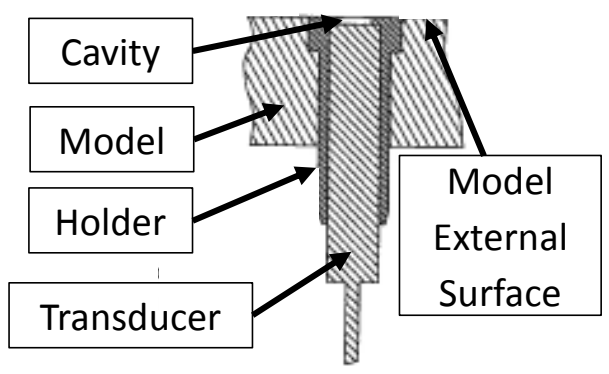

Figure 1. Transducer holder assembly. the transducer is mounted by way of a through-hole, gravity will tend to cause it to translate in its hole as the glue sets. Depending on the model's orientation, this translation means the transducer will either recess beneath or extend beyond the model outer surface. Either situation can artificially change the measured levels ${ }^{3}$. In the event of a transducer set proud to the model outer surface, machining can ensure flush mounting, but will almost certainly damage the transducer and diaphragm. Alternately, the transducer can be mounted directly to the inside of the model, where a through-hole smaller than the transducer diameter is located. Although this would ensure flush mounting without transducer damage, it creates a cavity that could induce noise. By this method, the cavity cannot be minimized without risk of damage to the model and becomes a problem of manufacturability.

In recent tests, the National Aeronautics and Space Administration (NASA) has solved the challenge of transducer flush mounting in aeroacoustic wind tunnel testing by use of special transducer holders. This holder, illustrated in Figure 1, is a sleeve into which the transducer fits, with a cap that allows the holder to be mounted in a recessed hole in the model. A single hole in the holder allows the transport of the tunnel flow medium so the transducer can discriminate the fluctuating pressure due to the turbulent boundary layer noise. The holder is first dry fitted into the model. The surface of the holder corresponding to the model external surface is sized such that it will always sit proud to the model surface. Any difference in height between the holder and the model surface can be machined and sanded flush, thus ensuring flush surface mounting. The holder is removed from the model after machining, and the transducer is glued inside the holder. Finally the holder/transducer assembly is replaced in the model and secured with glue. This methodology eliminates the problem of increases to measured fluctuating pressure level (FPL) due to lack of flushness while protecting the transducer from damage. Although a cavity results, as shown in Figure 1, this method of mounting allows for a smaller cavity than direct transducer mounting. In order to work with this holder design, special transducers have been procured with their standard screen removed and the diaphragm moved as close to the top of the casing as possible to minimize any cavity volume.

Although this greatly reduces induced noise due to the transducers being out of flush, the holders can also induce a cavity resonance that is usually at a very high frequency; around $40-60 \mathrm{kHz}$ model scale ${ }^{5}$ for air. This noise, termed transducer mount induced noise (XMIN), is generally of the same order of magnitude as the turbulent boundary layer induced noise, and so must be removed from the test data. However, the frequency of the resonance mode can vary with cavity depth, boundary layer noise that excites the mode, tunnel flow medium, angle of attack ( $\alpha$ ), and mean flow velocity. The internal shape and volume varies from transducer to transducer as well ${ }^{6}$, and so the cavity response varies from transducer to transducer. The variability of the XMIN, combined with the volume of transducers and test points required for flight vehicle environment characterization makes correcting the data for XMIN a significant challenge during data post-processing. The consequence is increased uncertainty and unquantifiable conservatism or non-conservatism in the resulting environments.

Because the boundary layer flow interaction with the transducer holder seems to affect the cavity resonance response, the NASA/George C. Marshall Space Flight Center (MSFC) aeroacoustics team postulated that changing the design of the holder might reduce the cavity resonance response. An experiment was performed at the MSFC Trisonic Wind Tunnel (TWT) comparing a new holder design with the traditional MSFC holder. The new holder design is intended to mimic the Kulite Semiconductors, Inc. B-screen, where the single hole of the traditional holder is replaced by a series of several smaller diameter holes. This design would serve to protect the transducer diaphragm as in the manufacturer's B-screen, while also increasing the frequency of the XMIN such that it is not apparent in the data collected from aeroacoustic wind tunnel tests. This paper presents the methodology and results of this experiment.

American Institute of Aeronautics and Astronautics 


\section{Model Design}

The MSFC Aerodynamic Research Facility Acoustic Calibration Cone \#416 was utilized for XMIN wind tunnel testing. The model consisted of a calibration cone, a sting adapter, standard MSFC transducer holders, and B-screen transducer holders. Figure 2 shows a diagram of the integrated model with the cone and sting bolted together.

\section{A. Calibration Cone}

The model used for this test is a 10 degree $\left({ }^{\circ}\right)$ calibration cone. The cone, illustrated in Figure 2, is 10 inches (") long, with five counterbored holes that can be used for transducer installation. Holes are located at 2.5", 5", and 7.5" from the tip along the $0^{\circ}$ face (labeled as holders $\mathrm{A}, 1$, and 2 respectively), and at $5 "$ and $7.5^{\prime \prime}$ from the tip along the $180^{\circ}$ face (labeled as holders 3 and 4 respectively). Both of these faces are flat rather than conical, with a 1.617" maximum separation between the flat faces. The maximum conical diameter is $1.75 "$ ". For this test, only holder locations 1, 2, 3, and 4 where utilized, while holder location A was filled with wax.

The cone is hollow to allow for instrumentation installation and to carry wiring and reference tubing through to the tunnel sting. Additionally, a notch is cut on the $180^{\circ}$ face to allow for easier access to the holder A location. This notch was filled with wax for testing. The cone also includes three static pressure ports. One port is located at 9 " from the tip on the $0^{\circ}$ face, and two are located at $8.25^{\prime \prime}$ and $9 "$ from the tip respectively on the $180^{\circ}$ face.

\section{B. Sting Adapter}

The XMIN test utilized a custom built 8.125" long sting adapter, which bolted to the back side of the calibration cone. The adapter was notched to correspond to a dowel at $0^{\circ}$ on the calibration cone, ensuring proper installation and orientation. The sting adapter is hollow to allow for instrumentation wiring and reference tubing to be routed from the calibration cone through to the tunnel sting. This sting is illustrated in Figure 2.

\section{MSFC Transducer Holder}

The standard MSFC transducer holder tested is a capped sleeve with a hollow inner diameter of sufficient size to just allow for the transducer to be inserted. The cap is of the same diameter as the counterbore of the holes in the model, allowing the transducers to be inserted in place without sinking into the model. This ensures that the holder will remain flush with the model surface during the gluing process. A single hole in the cap exposes the transducer diaphragm to the tunnel flow medium, allowing the transducer to measure the turbulent boundary layer noise. This hole is sized based on the transducer being used, where the hole is smaller in diameter than the transducer casing, but larger in diameter than the transducer diaphragm. The size of the resulting cavity is reduced to the smallest allowable volume while still ensuring the holder will retain integrity. The MSFC transducer holder dimensions must be tailored to the specific transducer model being utilized. The holder manufactured for this test is illustrated in Figures $3 \mathrm{a}$ and

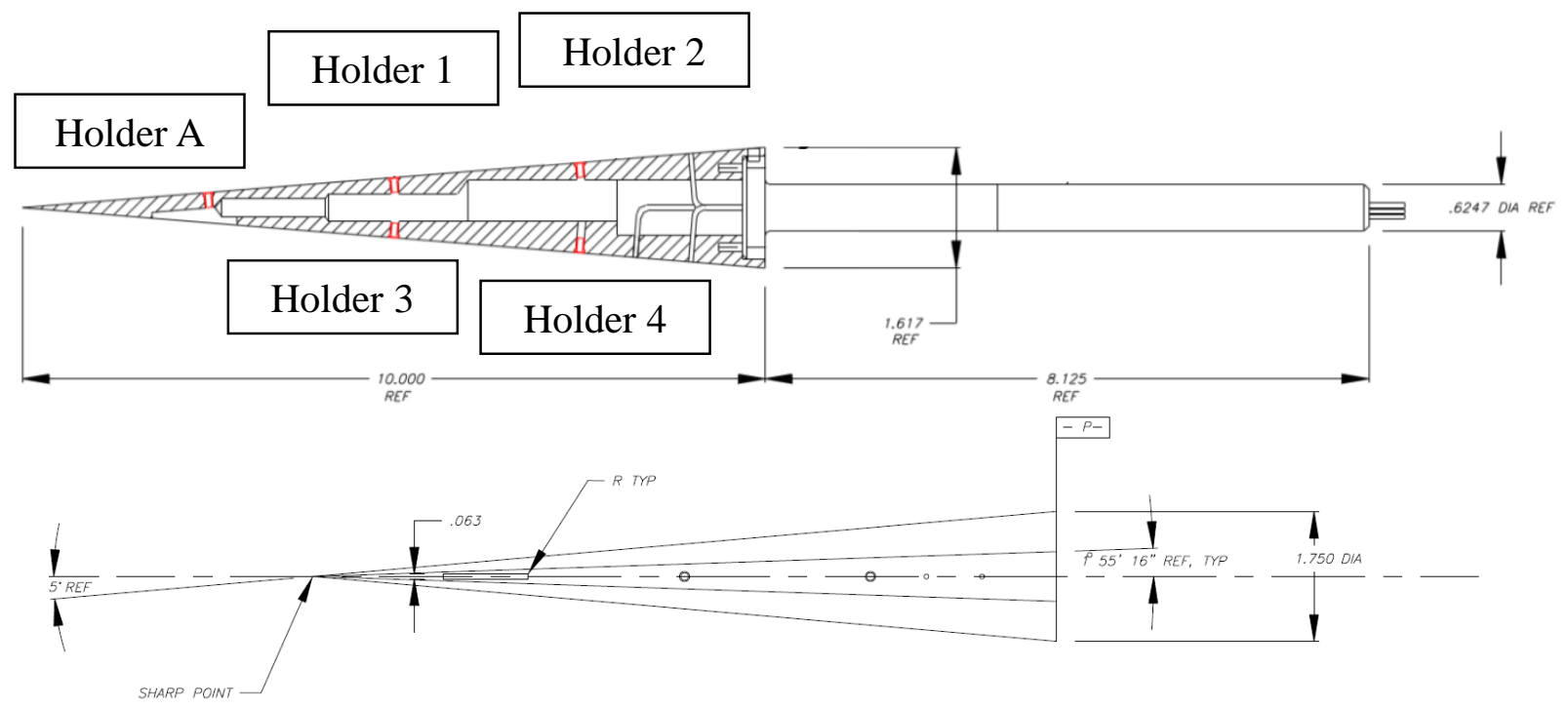

Figure 2. XMIN model and sting assembly (dimensions in inches). Holder port locations are shown in red.

American Institute of Aeronautics and Astronautics 
3b. Transducers were mounted using the traditional MSFC transducer holder in holder port locations 1 and 2 as seen in Figure 2.

\section{B-screen Transducer Holder}

The B-screen transducer holder was designed using the MSFC transducer holder as a template. The single hole was replaced with ten smaller holes arranged in a circle about the center of the holder. The diameter of this circle pattern was set using the same criteria as the single hole definition of the MSFC transducer, where the diameter must be less than the casing diameter of the transducer, but greater than the diameter of the transducer diaphragm. As with the traditional holder, the B-screen holder dimensions must be tailored to the specific transducer being utilized. The Bscreen transducer holder manufactured in this test is illustrated in Figures $3 \mathrm{a}$ and $3 \mathrm{c}$. Transducers were mounted using the B-screen transducer holder in holder port locations 3 and 4 as seen in Figure 2.

\section{E. Bowled B-screen Transducer Holder}

A variation on the B-screen holder was considered and manufactured, but ultimately not used. With this design, the cavity volume would be further reduced by replacing the flat internal surface of the cap with a convex internal surface. The challenge of the bowled B-screen holder design is that the diameter of the convex surface must be defined such that the surface does not contact the transducer diaphragm. This design was ultimately abandoned after transducer measurements showed that the variation in diaphragm depth from transducer to transducer in the chosen transducer model would make designing a universal holder design impossible ${ }^{6}$. This variation gave a high probability that a certain number of transducer diaphragms would be contacted by the convex internal cap and may be damaged or respond incorrectly to fluctuating pressure.

\section{Instrumentation}

\section{A. Transducers}

The transducer used for the XMIN test campaign was the XCL-20-IA-072-25D miniature high frequency fluctuating pressure transducer, manufactured by Kulite Semiconductor, Inc. This transducer was selected for several reasons. In recent unsteady aerodynamic wind tunnel test programs, NASA has used the XCL-072 series transducer with success, including the Ares I/Ares I-X ascent aeroacoustic tests and Space Launch System (SLS) rigid buffet model test. As a result, NASA personnel had experience and familiarity with the transducer, easing transducer integration in the XMIN test. Additionally, NASA chose the differential XCL-072 for the SLS ascent aeroacoustic testing. If the B-screen transducer holder design proved successful during the XMIN test campaign, it would be utilized for the SLS ascent aeroacoustic testing, making transducer commonality desirable.

As in previous tests, the transducers were ordered with their usual screens removed and the diaphragm cavity backfilled with room temperature vulcanized (RTV) silicone. This modification allows for the mounting of the transducer in a holder, while minimizing the transducer cavity. The transducer was further modified to reduce the length of the back end of the casing by $0.175 "$. This alteration allowed the transducers to better fit within the XMIN acoustic calibration cone while leaving the XCL-072 internal sensing diaphragm design unchanged.

These transducers were originally manufactured to a 15 pounds per square inch differential (psid) range, but were recalibrated by the manufacturer to 25 psid due to the dynamic pressures expected in the TWT. Each transducer had a reference tube which exited out the aft end of the sting. The transducers also featured an integrated amplifier and temperature compensation unit separated from the transducer by three feet of cable.

American Institute of Aeronautics and Astronautics 


\section{B. Data Acquisition System}

An eight channel Agilent Technologies system was utilized to collect alternating current coupled pressure data at a rate of approximately 196,000 samples per second. Four channels were used to collect the data from the four installed transducers. Because time stamping from the facility data acquisition system could not be easily mapped to the timing for the Agilent system, a fifth channel recorded the facility $\alpha$ measurement so that attitude changes within a given run could be mapped to the corresponding pressure time history. The remaining channels were unused. All other tunnel static measurements, including velocity and model roll $(\varphi)$, were recorded using the facility data acquisition system.

Both static and dynamic data were acquired during each run. Synchronization with the dynamic data system was accomplished by mapping sequence numbers in the static data system and the dynamic data system. Time history was mapped between both data acquisition systems using the Agilent system recorded $\alpha$. Manual operation of the dynamic system was required. Dwell time per point for each point in the test matrix was set for five seconds or longer where possible in order to maximize the amount of dynamic pressure data available.

\section{Transducer Checkout}

All transducers underwent a checkout process to confirm health before installation. During this process, a vacuum was placed on the diaphragm and held for five minutes to ensure diaphragm seal integrity. Transducer response with no pressure applied and with \pm 5 pounds per square inch (psi) applied was also recorded. The final health check was transducer response with a blast of compressed air blown across the transducer. All transducers responded as expected and were deemed healthy prior to testing.

\section{Transducer Installation}

Transducer installation was one of the most difficult tasks in this experiment due to the limited accessibility of the inside of the calibration cone and the small size and delicate nature of the transducer, its wiring, and reference tubing. Despite these challenges, only one of five transducers was damaged during transducer installation. To prepare for installation, each transducer holder was fitted into its respective holder location and sanded flush with the model surface. Prior to installation, each transducer reference tube was carefully bent so that the transducer assembly would fit inside the cone, with special effort made to prevent crimping the tube. The reference tube was then attached to additional tubing length sufficient to extend through the model, sting adapter, and sting. A piece of string was used to pull the transducer through the sting adapter and through the cone into its desired hole (Figure 4a). Once the transducer was pulled all the way through, it was glued into its holder in an orientation that would allow the reference tube to face back towards the sting adapter to prevent reference tube crimping. The transducers were then glued into their holders (Figure 4b) and the holder assembly was glued into the model (Figure 4c). Duco-Cement was used for both gluing steps. This glue was ideal because it is capable of withstanding the tunnel forces, and will release when exposed to acetone, allowing for removal of holders from the model and transducers from the holders for future testing or in the event that a transducer or holder needed to be replaced during testing.

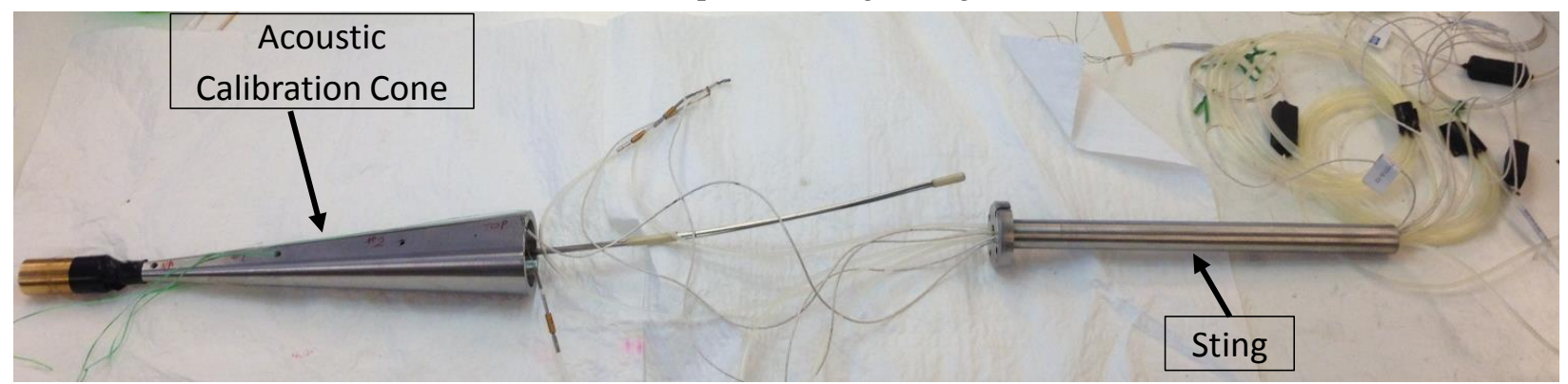

a.) Assembly prior to transducer and holder installation.

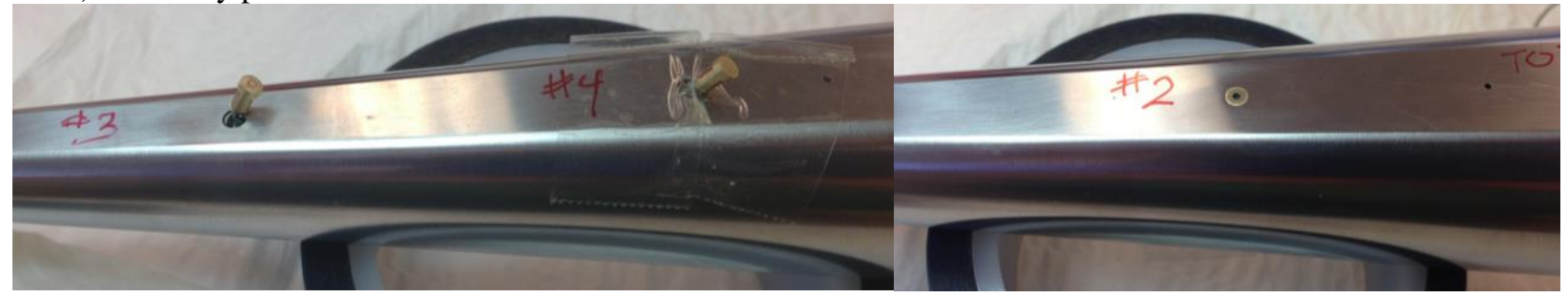

b.) Transducers secured in holders (B-screen shown).

Figure 4. Transducer installation into XMIN model and sting assembly.

American Institute of Aeronautics and Astronautics 


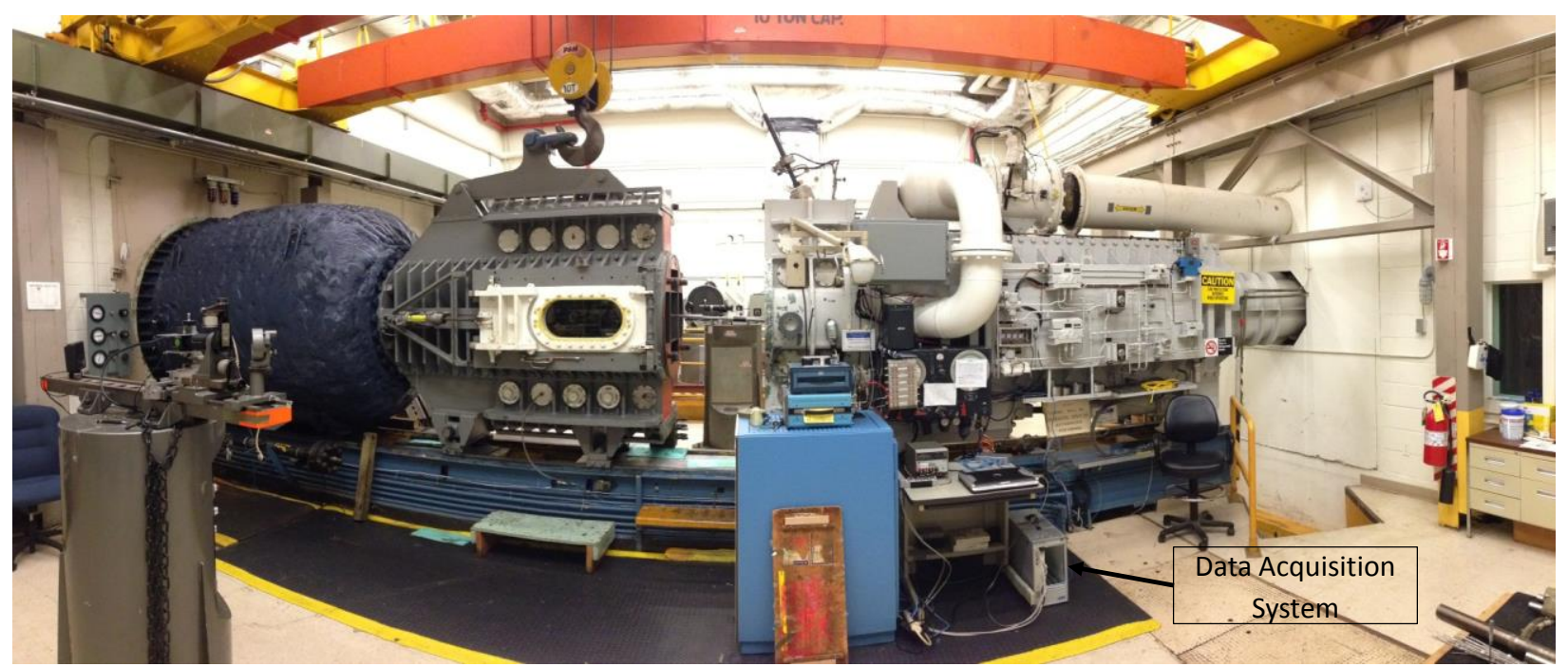

Figure 5. MSFC Aerodynamic Research Facility TWT (flow direction from left to right).

\section{Test Operations}

Testing for the XMIN experiment occurred over 11 - 13 February, 2013 and was designated by the facility as Test XP1.7. Over this period, 83 runs were obtained, during which 273 test conditions were measured. The primary objective of the test was to use the Kulite XCL-20-IA-072-25D transducers installed in both MSFC standard and Bscreen holders to collect data at a variety of conditions to determine if the B-screen holder would be sufficiently effective at reducing XMIN to justify the cost and schedule impact of using a more complicated to manufacture transducer holder in larger aeroacoustic tests. The success criteria set prior to testing was to collect useful data over a broad range of transonic Mach (M) numbers that would allow this objective to be met. Useful data were defined as data that could be transposed into frequency domain using Fast Fourier Transform (FFT) methodology. The success criteria were met, accomplishing all primary objectives.

\section{A. Test Facility}

The XMIN experiment was conducted in the MSFC Aerodynamic Research Facility 14 x 14-Inch TWT (pictured in Figure 5) at transonic and supersonic Mach numbers. This facility is an intermittent blowdown type wind tunnel facility with a 24" long test section capable of velocity conditions from Mach 0.25 to 5 using two interchangeable test sections and a series of nozzle sets. For the Mach ranges of interest for this experiment, typical run times were approximately 60 seconds ${ }^{7}$.

The XMIN acoustic calibration cone assembly was installed in the tunnel utilizing the TWT S6 extension sting, which centered the cone approximately in the middle of the tunnel test section. Tunnel station 20 corresponded to 3.125 " forward of the cone base. Figure 6 shows the XMIN model installed in the test section. All cabling, reference tubes, and static pressure tubes were routed internally through the cone assembly, stings, and then out through the tunnel wall. Transducer reference tubing was manifolded together and referenced to the tunnel plenum. Transducer cabling was connected to the high frequency data acquisition system, which was located immediately adjacent to the wind tunnel. The data acquisition system was controlled remotely from the control room. Model attitude of $\alpha, \varphi=\left\{0^{\circ}, 0^{\circ}\right\}$ was set using a level.

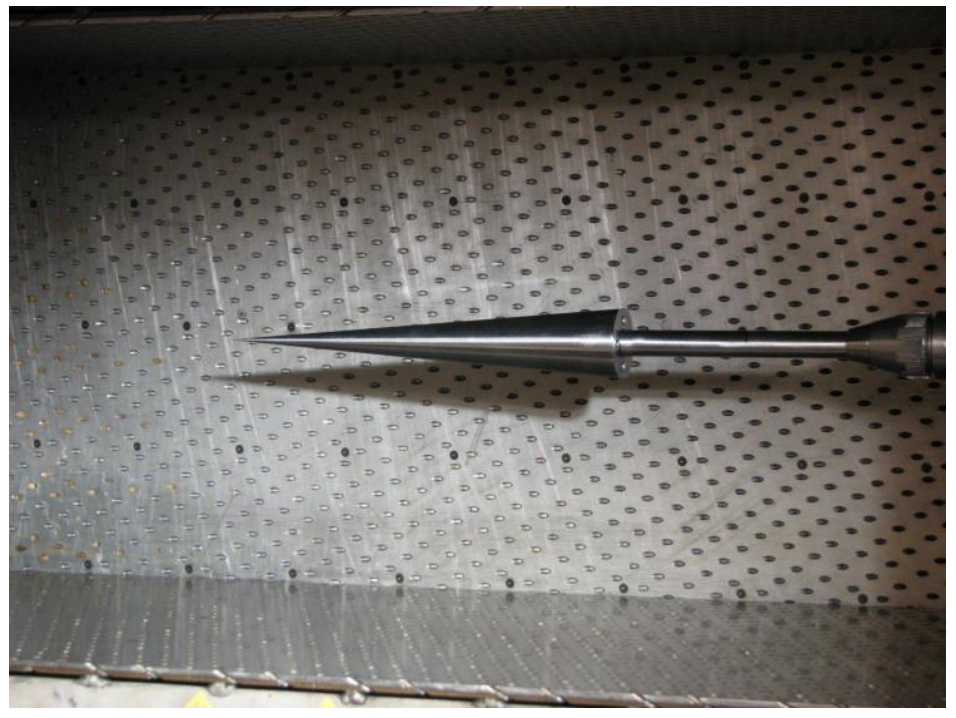

Figure 6. XMIN model installed in the TWT test section (flow direction from left to right). 


\section{B. Test Plan}

The test plan originally called for 104 total test conditions of varying velocity conditions, $\alpha / \varphi$ attitudes, and the addition of several flow trip methods ${ }^{8}$. Of these conditions, only a small subset were actually required to determine potential effectiveness of the B-screen holder. Each successive run after these initial conditions provided better understanding and confidence in the results.

Recent NASA aeroacoustic tests experienced transducer attrition rates of approximately three percent due to damage during model installation and configuration change, debris in the tunnel, and the delicate nature of the transducers. This normal attrition rate combined with pressures induced during the expected tunnel start-up and shutdown transients of the blowdown tunnel suggested a high probability of losing one or more transducers during testing. This test relied upon relative comparisons of a MSFC holder/B-screen holder pair to yield results useful for determining the effectiveness of the B-screen holder. Consequently, if one transducer in a pair was lost, no more data could be gained from that pair. If one transducer is lost from each pair, the test would need to be stopped, the model removed, and additional transducers obtained in order to progress further through the test matrix. Because this tunnel has not been used for dynamic pressure measurement in some time, there was uncertainty in the impact of normal tunnel operation on the transducers being used.

The matrix was therefore set to reflect the need and desire to collect certain sets of data before others in the event that one or more transducers were lost. Although this run order was not necessarily the most efficient means of tunnel operation to accomplish the matrix, it was the best way to satisfy the success criteria. The highest priority runs were those that collected data covering the full Mach range that required no block change in the tunnel $(\mathrm{M}=0.80$ through 1.30) and no $\alpha$ or $\varphi$ change. These conditions were deemed necessary and sufficient to make an engineering assessment of the viability of the B-screen holder. Because no change would be required to the tunnel or model physical configuration, opportunity for damage to the transducers due to model handling would be minimized. Each decrease in condition priority was set in place to increase confidence in the results found in those initial Priority 1 runs, while minimizing the added risk of transducer loss. Velocity conditions run for this block were $\mathrm{M}=0.80,0.90,0.95,1.00$, $1.05,1.10,1.15,1.20,1.25,1.30$. The test matrix was adjusted as testing progressed to add additional conditions as desired and to remove conditions that were deemed unnecessary.

Priority 2 runs repeated those Mach numbers at varying $\alpha$ to determine the holder impact during change in pitch. These attitude conditions were $\alpha=-2^{\circ},-1^{\circ}, 0^{\circ}, 1^{\circ}, 2^{\circ}$ for $\mathrm{M}=1.00$ and 1.25 . After examining these data, the attitude schedule for this priority was changed to $\alpha=-4^{\circ}, 0^{\circ}, 4^{\circ}$ because negligible difference in pressure spectra was seen at $\alpha= \pm 1^{\circ}$ and $\pm 2^{\circ}$.

For runs with pitch changes, the model was run using pitch-pause, where all $\alpha$ conditions in the pitch schedule were accomplished during a single run for each Mach condition, with maximum possible time at each $\alpha$. This allowed for measurements across varying flow fields. Sweeping through both positive and negative $\alpha$ allowed for comparisons between holders $1 / 2$ and $3 / 4$, where positive $\alpha$ for holders 1 and 2 is comparable to the same run at negative $\alpha$ for holders 3 and 4.

Priority 3 runs repeated those velocity and attitude conditions with the model rolled at $\varphi=90^{\circ}, 180^{\circ}$. Model roll was accomplished manually by rotating the calibration cone fixture clockwise. These runs were meant as a control to isolate any effects of the tunnel on the data. If there was a preferentially quiet or loud side of the tunnel, these runs would measure those differences in FPLs. Ruling out these tunnel wall effects would allow the data to show if a reduction in level was due only to the transducer holder being used.

Priority 4 runs repeated those velocity conditions at $\alpha=\varphi=0^{\circ}$ with the addition of a $0.090^{\prime \prime}$ tape layer placed midway between holders $1 / 3$ and $2 / 4$. The purpose of these runs was to determine the impact of a small protuberance on holder effectiveness. Priority 5 repeated the velocity conditions with the trip in place sweeping through the $\alpha$ schedule. Priority 6 would have repeated those conditions sweeping through the $\alpha$ and $\varphi$ schedules. However, because no indication was found in the data for tunnel orientation bias after the Priority 3 runs, these runs were deemed unnecessary and removed from the test matrix to save time and preserve the transducers.

When no discernable effect was seen using the tape layer trip during Priority 4 and 5 runs, a solder ring was added to provide a more significant protuberance. A third protuberance configuration was tried using a 0.120 " high aluminum ring. With this protuberance in place, $\alpha \leq-8^{\circ}, 8^{\circ}$ were run for $\mathrm{M}=0.80,0.90,0.95,1.30$ to identify any effects caused by extreme pitch angle.

Priority 7 runs were those requiring block changes both with and without protuberances installed. These velocity conditions were $\mathrm{M}=1.46,1.69,1.96$. As in Priority 6 , runs at $\varphi \neq 0^{\circ}$ were deemed unnecessary because no tunnel bias had been noted in Priority 3.

American Institute of Aeronautics and Astronautics 


\section{Analysis}

\section{A. Data Reduction}

FFT data reduction was accomplished using AI Signal Research Inc. PC Signal ${ }^{\circledR}$ signal analysis software. All comparisons were made in both power spectral density (PSD) and one-third octave FPL. NASA/MSFC typically provides its flight vehicle aeroacoustic environments in one-third octave FPL, and so examining results in this form would indicate how the B-screen holder design would affect environment releases ${ }^{2}$. PSDs are provided here because their examination gives results in fine resolution of the frequency domain. High frequency data were mapped to static tunnel data using run number and the $\alpha$ data recorded by the high frequency data acquisition system. Within a given run time history, the time range over which to perform PSD and one-third octave FPL analysis was determined and selected based on when the $\alpha$ time history arrived at steady state indicating the desired $\alpha$ attitude, and terminating before $\alpha$ time
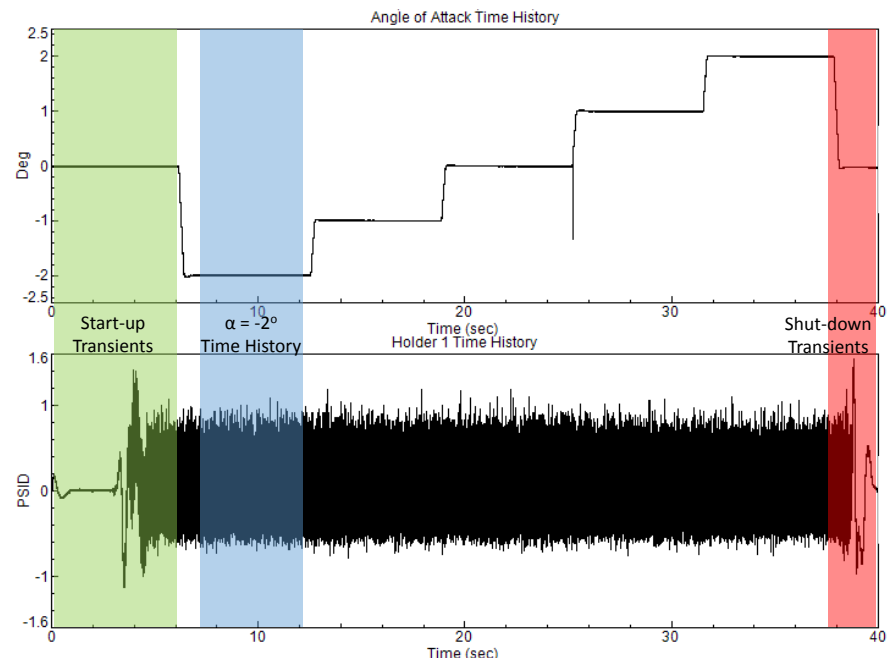

Figure 7. Example PC Signal ${ }^{\circledR}$ time history plot of $\alpha$ and pressure measured by the holder 1 transducer. history indicated the move to the next attitude in this schedule. This is illustrated with an example of $\alpha$ and pressure time history in Figure 7. As highlighted in blue in this example, $\alpha$ arrives at $-2^{\circ}$ at approximately seven seconds and dwells until roughly 12 seconds before moving to the next point in the attitude schedule. This gives approximately five seconds of pressure time history at $\alpha=-2^{\circ}$. For the first attitude point in a run, the time to begin sampling pressure data for analysis must be after the pressure history has reached steady state after the start-up transients. An example of the tunnel start-up transients are highlighted in green in Figure 7. Similarly, for the last point in a run, the time to end sampling pressure data must be before the pressure history indicates the shut-down transients. An example of the tunnel shut-down transients are highlighted in red in Figure 7.

Actual sample rate achieved was 196,608 samples per second. PSDs and one-third octave FPL analysis used a Nyquist cutoff of 2. All data were examined in model scale, employing no frequency or amplitude scaling. Manufacturer provided calibration data for each individual transducer was used for data analysis.

For comparisons between transducers at $\alpha=0^{\circ}$, holder 1 could be directly compared to holder 3 at a given velocity condition, as both transducer holders should see an equivalent external environment. Similarly, holder 2 could be directly compared to holder 4 . For appropriate comparison between transducers at $\alpha \neq 0^{\circ}$, direct comparisons were made at the relative attitude experienced by the individual transducers within a pair in order to ensure equivalent flow field. For example, to appropriately compare holder 1 and holder 3 at $\alpha=4^{\circ}$, data for holder 1 at $\alpha=4^{\circ}$ must be compared to data for holder 3 at $\alpha=-4^{\circ}$, where each transducer sees flow approximately parallel to the holder surface.

\section{B. Data Quality}

The noise frequency content of the tunnel was unknown going into the test campaign, and so the impact of that noise as compared to the turbulent boundary layer noise caused by the model was also an unknown. However, this test is designed to measure the differences in magnitude between the traditional MSFC and proposed B-screen holder designs. Measurement of the model external aeroacoustic environment itself is not necessarily important so long as XMIN is discernable above the tunnel noise.

Data quality was assessed primarily through data comparison. Repeat runs at $\alpha, \varphi=\left\{0^{\circ}, 0^{\circ}\right\}$ were added at the end of the test matrix for Machs 0.80 through 1.30 to ensure consistent data measurement throughout the entire test campaign. These conditions were also run at $\varphi=0^{\circ}, 90^{\circ}$, and $180^{\circ}$ throughout the test campaign because $\alpha=0^{\circ}$ was included in all pitch schedules. All conditions run at $0.80 \leq \mathrm{M} \leq 1.30$ and $\alpha, \varphi=\left\{0^{\circ}, 0^{\circ}\right\}$ had at least five repeats, with at least one repeat run on each day of testing. These comparisons demonstrate good data quality and transducer health throughout the test campaign. Figure 8 provides an example of PSDs of repeat runs at M, $\alpha=\left\{0.80,0^{\circ}\right\}$ across runs $10,29,38,49$, and 85 , which include $\varphi=0^{\circ}, 90^{\circ}, 180^{\circ}$. The repeatability evident in this figure is consistent across all $\mathrm{M}$ conditions tested.

If the B-screen holder transducers gave reduced levels as compared to the MSFC holder transducers, it was crucial to ensure that the drop in level was due entirely to the change in holder design. However, because each face of the

American Institute of Aeronautics and Astronautics 
model held the same type of transducer, if one side or section of the tunnel was preferentially quieter than another, this could cause a false positive. By repeating $M$ and $\alpha$ runs at different $\varphi$, any differences or biases that may be due to a particular side of the tunnel can be determined. Tunnel side biases were ruled out based on these runs. As seen in Figure 8, no discernable tunnel orientation bias is seen through the $\varphi$ schedule.

Additional health spot checks were performed to ensure data were as expected for this type of test. Time histories indicated pressures of the correct order of magnitude and gave no signs of clipping due to sensor over-ranging. Histograms of pressure time histories confirm the data to be Gaussian distributions with minimal skewness. Also, data collected with the tunnel off showed consistency between transducers throughout the test campaign.

Although calibration checks were not performed after transducer installation, repeat runs throughout the test, tunnel off measurements, and

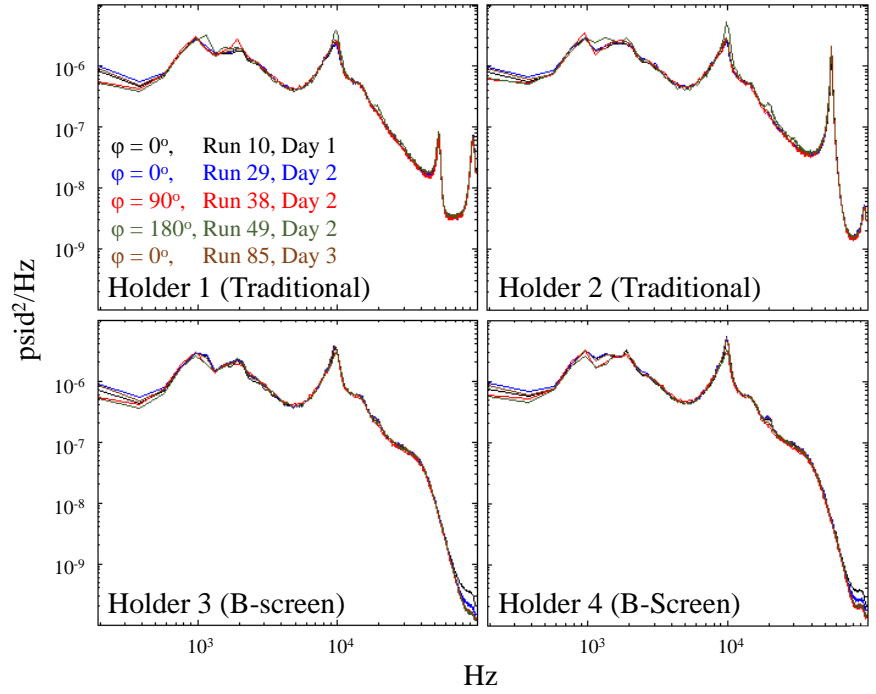

Figure 8. Repeat runs at $M, \alpha=\left\{0.80,0^{\circ}\right\}$ and $\varphi=0^{\circ}, 90^{\circ}$, $\mathbf{1 8 0}^{\circ}$ for all transducers (Runs $\left.10,29,38,49,85\right)$. time history and histogram examinations prove transducer health was maintained throughout testing. All XMIN runs were completed without loss or degradation of any of the four transducers based on these metrics.

A review of the test, methodology, analysis, and results was held on 26 February 2013 at Langley Research Center, including participants from the Ames Research Center, Johnson Space Center, Langley Research Center, MSFC, and the NASA Engineering Safety Council as part of the SLS Aerodynamics technical review process.

\section{Results}

General flow characteristics observed in the test results are summarized in Figures 9 and 10 and are as expected for the facility, model, and holders. Frequency in these plots are left on a linear scale to better highlight the frequency of XMIN as opposed to the logarithmic scale that is generally used for aeroacoustic study. For better resolution below $10 \mathrm{kHz}$, see Figure 8.

Several features described as tunnel noise are evident in the data for all Mach and attitude conditions tested. Dominant peaks occur at roughly $1 \mathrm{kHz}$ and $10 \mathrm{kHz}$. As seen in Figure 9, these peaks do not move significantly in frequency content throughout the Mach regime tested. Figure 10 shows that they do not shift significantly with change in angle of attack. These data suggest that the $1 \mathrm{kHz}$ and $10 \mathrm{kHz}$ peaks are caused by the tunnel rather than by the model. Harmonic peaks associated with these noise sources are also discernable, with amplitude increasing with Mach until the supersonic block change occurred. With this change, tunnel noise and harmonics continued to dominate with similar shape but with a lower amplitude. Change in tunnel induced noise is expected with a change in nozzle shape. These data indicate that tunnel noise dominates model induced FPLs, especially in the frequencies below $20 \mathrm{kHz}$. Tunnel noise sources include the tunnel control valve, nozzle throat, and porous walls ${ }^{9}$.

Although results in Figure 9 are only at $\alpha, \varphi=$ $\left\{0^{\circ}, 0^{\circ}\right\}$ for $\mathrm{M} \leq 1.05$, relative trends are consistent across all $\alpha$ and $\varphi$ tested. All measurements for M $\geq 1.10$ are consistent in shape and trend to PSDs for $\mathrm{M}=1.00$ and 1.05 in Figure 9 and have been left out to simplify the plot. These data suggest that for $\mathrm{M} \leq 0.95$, all transducers may measure turbulent boundary layer noise induced by the calibration

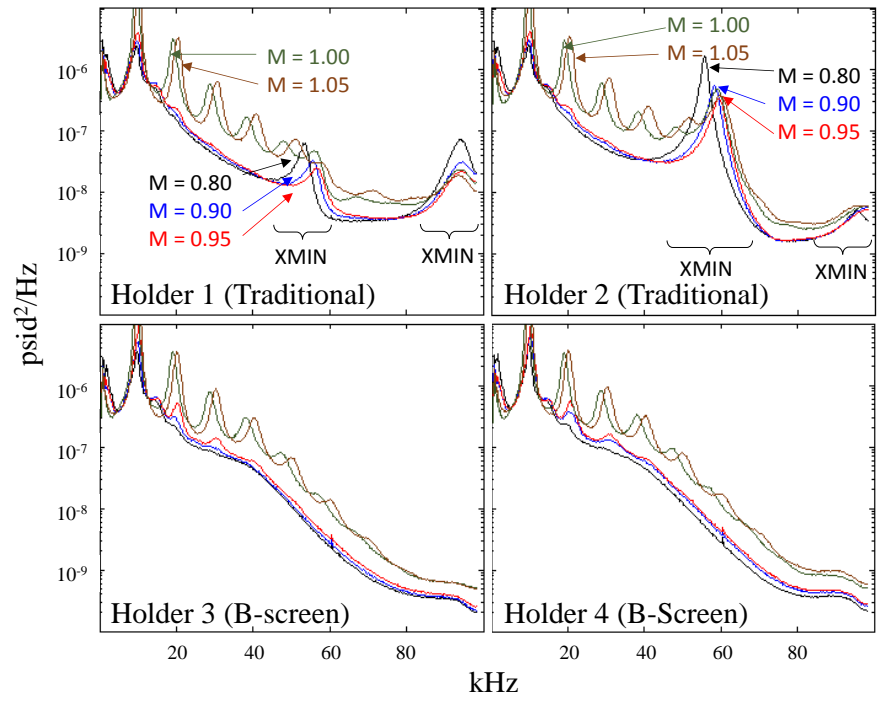

Figure 9. Runs at $\alpha, \varphi=\left\{0^{\circ}, 0^{\circ}\right\}$ for $M=0.80,0.90,0.95,1.00$, 1.05. $M \geq 1.10$ data are consistent with $M=1.00,1.05$. 
cone above $20 \mathrm{kHz}$. However, once tunnel conditions reach $\mathrm{M}=1.00$, tunnel noise and associated harmonics definitely dominate, and remain dominant through supersonic measurements.

Another dominant feature in these data is a tone that is only clearly discernable in the MSFC holder measurements (holders 1 and 2), especially in the transonic Mach numbers. Above $\mathrm{M}=1.00$, the peak becomes less evident with increase in Mach number as tunnel noise becomes increasingly dominant. This peak appears at approximately 55 $\mathrm{kHz}$, and proves more dominant in amplitude in holder 2, located towards the aft end of the model. Additionally, a first harmonic is evident. Because this tone is almost completely unseen by the Bscreen holder measurements (holders 3 and 4) in all runs, this tone can be identified as XMIN. Additionally, this tone appears in the frequency range where XMIN is reported by recent larger

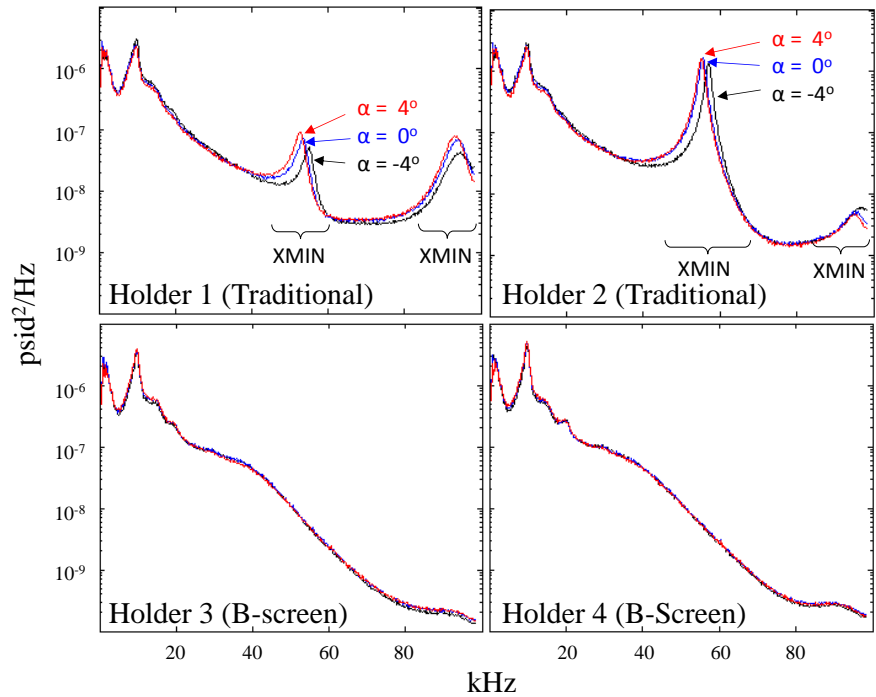

Figure 10. $M=0.80$ for $\alpha=-4^{\circ}, 0^{\circ}, 4^{\circ}$. scale model wind tunnel tests utilizing an identical transducer series and standard transducer holder. Because the XMIN peak is most prominent for $\mathrm{M} \leq 0.95$, these runs prove the most illustrative for the purposes of this experiment, with the best being at $M=0.80$. Although $M \geq 1.00$ conditions in the TWT may not be appropriate for measuring turbulent boundary layer noise with this particular model, comparisons between the transducer holder pairs still demonstrate consistent B-screen holder effectiveness in the supersonic regime.

This experiment shows that XMIN amplitude and frequency are affected by Mach and attitude tested, which is consistent with experience. As in Figure 9, XMIN decreases in amplitude and increases in frequency with increase in Mach. Figure 10 shows a similar effect when varying $\alpha$. Although results are provided only at $\mathrm{M}=0.80$, relative trends are consistent across all Mach numbers tested. The MSFC holder transducers measure XMIN most strongly at positive angles of attack. These changes may possibly be explained by a change in damping and effective cavity size due to the degree of flow impingement on the cavity itself. This would occur with an increase in Mach and also where the model is at $-\alpha$ with respect to the transducers. However, the phenomenon driving XMIN is not yet well understood due to the size and geometry of the transducers, the variability from transducer to transducer, the effects of the RTV, and other unknown factors. A proven explanation of these minor frequency and amplitude changes with Mach and attitude is outside the scope of this experiment. Regardless of the exact mechanisms at play, the results of this experiment are sufficient to show that while the MSFC transducer holders did induce what can be described as XMIN, the B-screen holders were able to minimize the presence of XMIN without negatively impacting data at other frequency bands.

Although Figures 8, 9, and 10 show that the MSFC holder transducers measure XMIN and the B-screen holders measure minimal XMIN with a perceptible but muted first harmonic as compared to the single hole holder, a more direct comparison is instructive for determining actual effectiveness of the B-screen holder. In order for the B-screen to be an appropriate solution, it must reduce XMIN while not negatively impacting actual model noise measurement. Figure 11 provides such a comparison for $\mathrm{M}=0.80$ where $\mathrm{XMIN}$ is most pronounced. Although the remaining velocity conditions are not provided here, the trends are consistent through the remaining velocity and attitude conditions tested.

Figure 11 gives good agreement between MSFC and B-screen holders below $40 \mathrm{kHz}$, suggesting that the B-screen holder does not interfere with measurement of noise phenomena

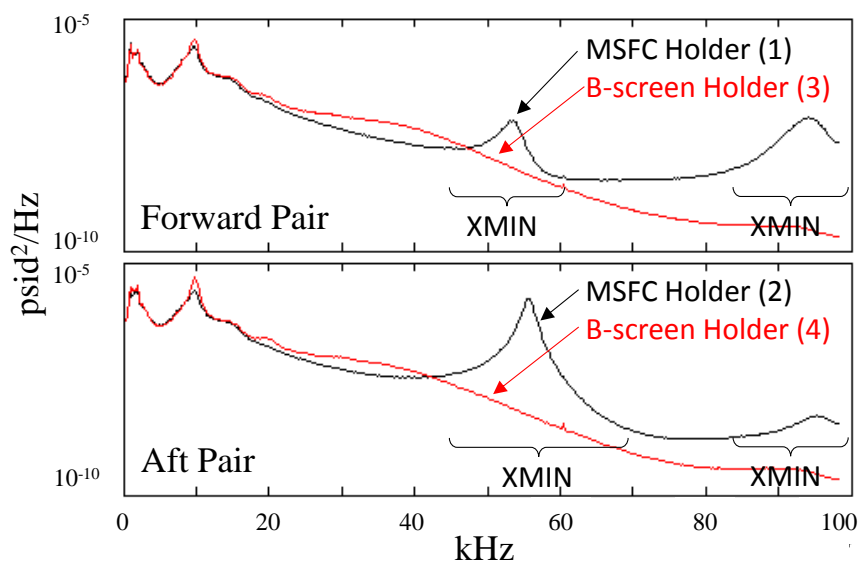

Figure 11. $M, \alpha=\left\{0.80,0^{\circ}\right\}$ comparison between traditional MSFC and B-screen holders for forward and aft pair.

American Institute of Aeronautics and Astronautics 
external to the transducer holder. Approaching the XMIN frequency, the B-screen holders clearly minimize the impact of the XMIN seen in the MSFC holder data. Additionally, the B-screen holder transducers measured a negative slope in the spectra as frequency increases as would be predicted, in contrast to the near zero or positive slope seen beyond the XMIN peaks in the MSFC holder. Similar trends are seen through the supersonic velocity runs. Although the XMIN peak was not clearly seen above $\mathrm{M}=1.00$, the $\mathrm{B}$-screen holder measurements showed a continued negative slope above $40 \mathrm{kHz}$ as would be expected, while the MSFC holders show a slope of near zero. B-screen holders proved effective for protuberance runs as well as non-protuberance runs.

\section{Conclusion}

The XMIN test campaign resulted in data collection over a broad range of transonic Mach numbers and attitude conditions. The data collected were transposed into frequency domain via FFT and compared for understanding. As such, the XMIN test campaign was a success as laid out by the test success criteria. Data reveal that distinguishable XMIN was stimulated by the MSFC holders. Additionally, these data show that the B-screen holder is effective at minimizing XMIN without reducing externally driven noise as compared to the MSFC holders. Although additional testing would be required to increase confidence in this conclusion, sufficient evidence was presented to motivate the use of the B-screen holder for large scale aeroacoustic wind tunnel testing with minimal risk to data collection.

As a result of these data, NASA/MSFC decided to implement the use of the B-screen holder design for all primary measurements in the SLS ascent aeroacoustics wind tunnel test, conducted at the Ames Research Center Unitary Plan Wind Tunnel in August and September 2013, with additional entries in December 2013 and January 2014. This test successfully utilized several hundred of the B-screen holders and showed minimal XMIN in the data collected. Several transducers on this test were mounted using the traditional MSFC single hole design and were co-located with Bscreen holder mounted transducers. Comparisons between data from these transducers, as well as with co-located transducers from previous SLS wind tunnel tests utilizing the traditional MSFC holder design agree well, giving further confidence in the use of this new B-screen holder design. The reduction in data processing effort and increased confidence in data in the highest one-third octave FPL bands of interest for the SLS ascent aeroacoustics test justified the added cost of B-screen holder manufacturing.

\section{Acknowledgments}

The authors would like to acknowledge the MSFC/ET20 and MSFC/ET10 wind tunnel test team, including Holly Walker, James Aaron, Bo Jones, Derek Mayer, Mark Perry, Richard Norman, and Alvin Mayers. Special thanks are owed to James Babicz of MSFC/ET20 who was responsible for transducer installation. The authors would also like to acknowledge Sandeep Patel, formerly of MSFC/ER42 and currently of Patel Technologies Corporation for data acquisition setup and support. Additionally, Chip Moore of MSFC/EM10 and Richard Norman of MSFC/ET20 deserve special recognition for solving the challenge of measuring the internal dimensions of the XMIN test transducers with high precision using non-destructive means.

\section{References}

1 “Ares I Acoustic Environments Data Book,” CxP 72164, 2009.

2“Dynamic Environmental Criteria NASA Technical Handbook,” NASA-HDBK-7005, March 2001.

${ }^{3}$ Efimtsov, B. M, et. al., "Effect of Transducer Flushness on Measured Surface Pressure Fluctuations in Flight," 43 ${ }^{\text {rd }}$ AIAA Aerospace Sciences Meeting and Exhibit, CP 2005-0800, AIAA Meeting Papers on Disc [CD-ROM], Disc 2, AIAA, Reston, VA, 2005.

${ }^{4}$ Robertson, J. E., Slone Jr., R. M., and Wang, M. E., "Study to Define Unsteady Flow Fields and Their Statistical Characteristics," NASA-CR-144092, 1975.

${ }^{5}$ Nance, D. K., “Aeroacoustic Environment Development via Experimental Techniques,” MSFC/EV33 Constellation Archive, NASA/George C. Marshall Space Flight Center, Alabama, 2010.

${ }^{6}$ Moore, L. E., Norman, R. R., "Kulite Transducer Multi-focus Z-Stack Images Using Leica MZ16A Optical Stereo Microscope and Depth Measurement Using Vertical Scanning Interferometry (VSI)," MSFC/EV33 XMIN Archive, NASA/George C. Marshall Space Flight Center, Alabama, 2012.

${ }^{7}$ Simon, E., "The George C. Marshall Space Flight Center's 14 x 14 In. Trisonic Wind-Tunnel Technical Handbook," NASA TM X-64624, Nov. 1971.

${ }^{8}$ Herron, A. J., Reed, D. K., Crosby, W. A., "Pre-Test Plan For: Transducer Mount Induced Noise (XMIN) Wind Tunnel Test, Facility Test Number XP1.7," MSFC/EV33 XMIN Archive, NASA/George C. Marshall Space Flight Center, Alabama, 2013.

${ }^{9}$ Schutzenhofer, L. A., Howard, P. W., "Suppression of Background Noise in a Transonic Wind-Tunnel Test Section," AIAA Journal, Vol. 13, No. 11, 1975, pp. 1467-1471.

American Institute of Aeronautics and Astronautics 